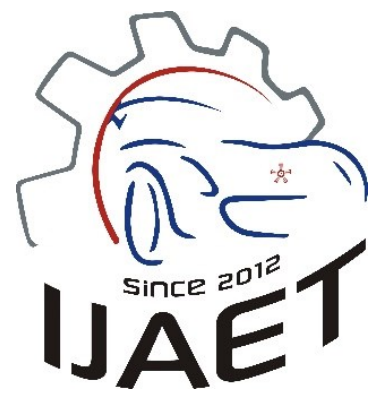

e-ISSN:2146 - 9067

International Journal of Automotive Engineering and Technologies

journal homepage: http://ijaet.academicpaper.org

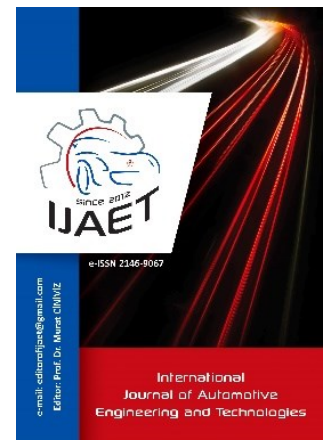

Original Research Article

\title{
Effects of under body diffuser on the aerodynamics of a generic car
}

\author{
Seyfettin Bayraktar, ${ }^{1, *}$, Yllmaz Ogun Bilgili ${ }^{2}$ \\ ${ }^{1}$ Yildiz Technical University, Department of Naval Architecture \& Marine Engineering, Istanbul, Turkey \\ ${ }^{2}$ Norse Design Engineering, 34947, Istanbul, Turkey
}

\author{
ARTICLE INFO \\ * Corresponding author \\ sbay@yildiz.edu.tr \\ Received: June 03, 2018 \\ Accepted: August 13, \\ 2018 \\ Published by Editorial \\ Board Members ofIJAET \\ (C) This article is \\ distributed by Turk \\ Journal Park System \\ under the CC 4.0 terms \\ and conditions.
}

\begin{abstract}
Flow structure and other aerodynamic properties such as drag and lift coefficients of one of the extensively investigated generic cars so-called Ahmed body are reported in the present numerical study for the fixed ground and upswept rear. For this purpose, Ahmed body with the slant angle of $25^{\circ}$ is considered and its rear bottom edge is upswept with various angles from $5^{\circ}$ to $30^{\circ}$ by $5^{\circ}$ increments without any side plates. It is observed that the sizes of the upper and lower recirculation bubbles seen in the profile section are decreased compared with the classical Ahmed body, however, the existence of some additional recirculation bubbles are observed when the upswept angle increases. At the highest upswept angle, the upper and bottom vortices called counter-rotating vortices (CVP) become nearly symmetric in the nearfar region however they merge and only one CVP forms behind the body in the far-field. Effects of the upswept angles on the drag and lift coefficients reveal that drag coefficient reduces the upswept angle to $15^{\circ}$ and then rises at $20^{\circ}$ while lift coefficient increases after $25^{\circ}$.
\end{abstract}

Keywords: Computational fluid dynamics (CFD); Ahmed body; Ground vehicles; Turbulence; Recirculation bubbles

\section{Introduction}

There has recently been renewed interest in finding new technologies to reduce aerodynamic drag and hence improve fuel consumption in the automotive industry because of the continuing high fuel costs in the past few years as well as the issue of global warming. It was reported that aerodynamic drag is responsible for more than $50 \%$ of the propulsive fuel consumption of a typical car running on the highway [1].

Road vehicles are bluff bodies in very close proximity to the ground. Their detailed geometry is extremely complex. The flow over a vehicle is $3 \mathrm{D}$ and their boundary layers are fully turbulent. Flow separation is common and followed by reattachment(s). Large turbulent wakes are formed at the rear and in many cases contain longitudinal trailing vortices. Furthermore, skin friction is negligible and the aerodynamic forces such as drag and lift forces are mainly governed by pressure losses. Among various road vehicles, an automobile has a streamlined shape at the front, but an angulated bluff shape at the back. This design causes highly complex and abstruse rear flow 
patterns, such as eddies, vortices, up-wash, downwash and mixed wake flows. Therefore, controlling the rear flow of an automobile is a core technology that can improve the aerodynamic performance. Due to these reasons, numerous studies have been performed to reduce automotive aerodynamic drag via passive or active flow controls of the rear wake of a car [2]. Changing the shape of the body or using some add-on devices are one of the most investigated approaches to achieve such goals.

In 1984, ref. [3] used a generic car called Ahmed body which is perhaps the most widely studied simplified car model in the automotive industry. Several experimental and numerical studies on the Ahmed body indicate three major components in the wake of this body: A recirculation bubble over the slanted surface or rear window, one pair of counter-rotating longitudinal or C-pillar vortices originating from the two side edges of the rear window, and a recirculation torus behind the vertical base. Aerodynamic drag is directly linked to the interactions between these three types of coherent structures. It was proposed a very simple and easily evaluative model with a cubical shape that no longer really looks like a car but reproduces its global behavior: a large 3D displacement in the front, a relatively uniform flow in the middle, and a large structured wake at the rear [3]. As reported later, the rear end is a simplification of a socalled fastback one such as on a Volkswagen Golf I [4].

Using different combinations of moving flaps that were fixed on every edge around two rear flat surfaces of a modified Ahmed body to control the flow was investigated [5]. It was reported that using flaps reduced drag $25 \%$ and lift $105 \%$, respectively. It was experimentally investigated the effects of shape on aerodynamic forces and moments of a $1 / 5^{\text {th }}$ model of a mini-van type vehicle under the effects of side flows [6]. It was showed that small variations of the A-pillar radius have an important effect on the side-force and yawing moment. Using a line of non-conventional trapezoidal vortex generators, a passive flow control technique was applied to a modified Ahmed body [7]. It was reported that drag and lift force on the body was reduced by $12 \%$ and
$60 \%$, respectively and the largest drag and lift reduction is clearly associated to a strong increase of the size of the recirculation bubble over the rear slant. Investigation of the velocity field in a cross section downstream the model reveals that the intensity of the longitudinal trailing vortices is strongly reduced and the drag reduction is due to the breakdown of the balance between the separation bubble and the longitudinal vortices. As another type of passive flow control devices, a deflector was placed on the upper edge of the Ahmed model's rear window [8]. Depending on the deflector angle $9 \%$ in drag reduction was achieved because the deflector increased the separated region on the rear window and let disruption the development of the counterrotating vortices appearing on the lateral edges of the rear window. Unlike the previous passive drag reduction techniques, rear-end periodic forcing as flow control method on an Ahmed body model was used [9]. Depending on the type and location of pulsed jets actuation, the maximum drag reduction (6 8\%) was obtained for increasing injected momentum or well-defined optimal pulsation frequencies. One array of circular cylinders upstream of the upper edge of the rear window of Ahmed body was deployed to suppress of the separation region near the upper edge of the slanted surface and increase the strength of the C-pillar vortices near the side edges of the rear window which led to longer separation bubbles behind the vertical base, producing a significant drag reduction by $11.6 \%$ [10]. It was experimentally investigated the effects of an array of cylindrical vortex generators on a $25^{\circ}$ slanted rear end Ahmed body at $\mathrm{Re}=1.35 \times 10^{6}$. It was reported that $10 \%$ in the overall drag reduction was achieved due to the suppression to the re-circulation bubble [11].

To decrease the drag and increase the lift coefficient a new design is proposed in the present study. For this purpose, the intersection of the bottom surface and the rear of the body is upswept with various angles and their effects are investigated numerically in terms of drag and lift coefficients for various Re numbers.

\section{Computational Details}

Ahmed body has been largely used as a reference model for both experimental and 
numerical investigations due to its simplicity. The main dimensions of the Ahmed body are given in Fig.1. The total length (L) of the body is $1.044 \mathrm{~m}$ from front to end. It is $0.288 \mathrm{~m}$ in height $(\mathrm{h})$ and $0.389 \mathrm{~m}$ in width. Cylindrical legs (strut) $0.05 \mathrm{~m}$ in length are attached to the bottom surface. Although Ahmed body has been investigated for various slant angles, the body with the slant angle of $\alpha=25^{\circ}$ is considered in the present study for validation process before its lower end is upswept with various angles to obtain a diffuser-like device.

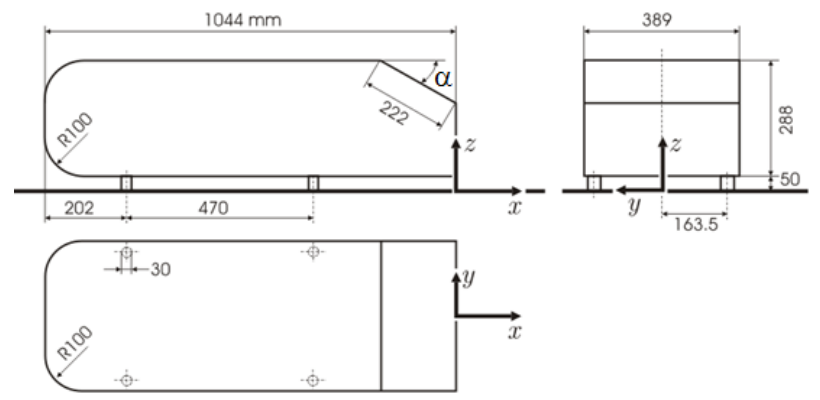

Figure 1 Main dimensions of generic Ahmed body.

In the aerodynamic of the ground vehicles, diffusers are used to convert the kinetic energy of the flow into the pressure rise by increasing the flow cross-sectional area. A diffuser is formed by a channel between an upswept surface and the ground. They are generally used at the rear of the vehicles in order to generate downforce by slowing down the low pressurized but accelerated flow from the underbody of the vehicle into a highpressurized and hence increases velocity in order to reduce lift and accordingly minimize the drag forces. The devices, therefore, generate downforce over the vehicle. It is designed as the closed channel on both sides by end-plates or side plates between an upswept aerodynamic surface and the ground and acts as an expansion chamber [12]. Furthermore, the diffuser also smoothes out the airflow at the back, reducing drag. In the present study, validation processes are done for Ahmed body with the slant angle of $\alpha=25^{\circ}$. After the validation process, some modifications were done on the body by raising the lower end of the rear side with various angles from $\beta=5^{\circ}$ to $30^{\circ}$ by $5^{\circ}$ increments (Fig. 2 ).

Ahmed bodies with different upswept angles are located in a virtual wind tunnel called computational domain in dimensions of
$10 \mathrm{~L} \times 3 \mathrm{~L} \times 3 \mathrm{~L}$ in the stream-wise, span-wise and normal directions, respectively where $\mathrm{L}$ stands for the total length of the body. The size of the domain gives a blockage ratio of $1.14 \%$. Computational domain starts at two body length (2L) in front and 6L behind the Ahmed body (Fig.3). Center of the coordinate system was placed at the end of the body $(x=0$ end of the body, $\mathrm{y}=0$ symmetric plane, $\mathrm{z}=0$ ground plane). The symmetry boundary condition was applied since the body is symmetric in $\mathrm{x}-\mathrm{Z}$ plane. The Ahmed body and the ground are kept fixed during analyzes.

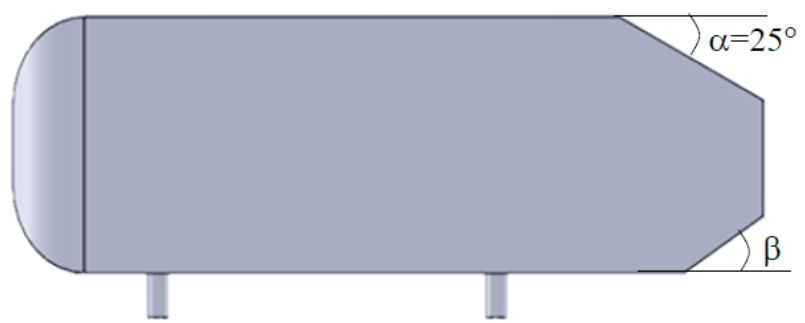

Figure 2 Definition of slant $(\alpha)$ and upswept $(\beta)$ angles of Ahmed generic car.

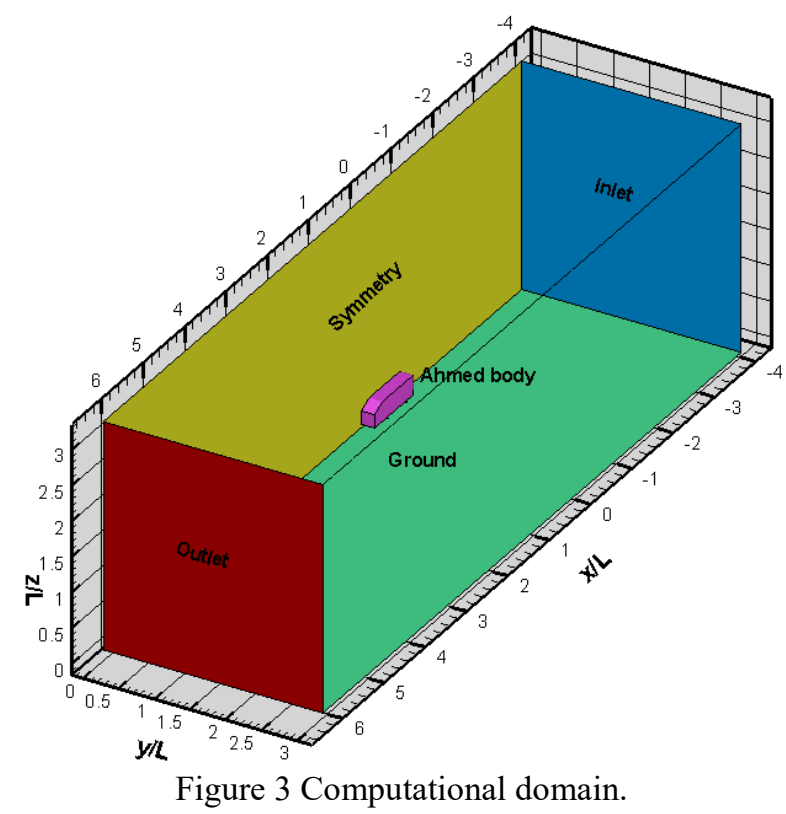

The no-slip condition is applied to the surfaces of the Ahmed body and the ground while uniform velocity $\left(\mathrm{u}_{\infty}\right)$ and the pressure outlet are imposed to the inlet and outlet of the computational domain, respectively. Symmetry boundary condition is used for the top and back of the domain. Analyses are performed for various Re numbers as shown in Table 1.

Estimation of the aerodynamic properties of a solid body is strongly affected by the grid resolution on the body, therefore, a boundary layer mesh is applied to the body, and then 
Ahmed body is enclosed by a small sized box to apply very small mesh elements around it as shown in Fig.4. Approximately 1.5 million mesh elements are generated by increasing its density toward the body. Since the flow in the boundary layer affects the aerodynamic properties of the body, a structural mesh is applied this region while unstructured mesh is applied to the far field regions. Special attention is not only paid to the boundary layer over the body but also to the walls of the computational domain.

Table 1. Parameters and their magnitudes used in the simulations.

\begin{tabular}{lll}
\hline Parameter & Magnitude & Description \\
\hline $\mathrm{L}(\mathrm{m})$ & $1.044 \mathrm{~m}$ & Body length \\
$\mathrm{H}(\mathrm{m})$ & 0.288 & Body height \\
$\mathrm{u}_{\infty}(\mathrm{m} / \mathrm{s})$ & $10,20,30,40$ & Freestream velocity \\
$\operatorname{Re}(-)$ & $0.71 \times 10^{6}, 1.42 \times 10^{6}, 2.13 \times 10^{6}, 2.85 \times 10^{6}$ & Reynolds number \\
$\alpha\left(^{\circ}\right)$ & 25 & Slant angle \\
$\beta\left({ }^{\circ}\right)$ & $0,5,10,15,20,25,30$ & Upswept angle \\
\hline
\end{tabular}

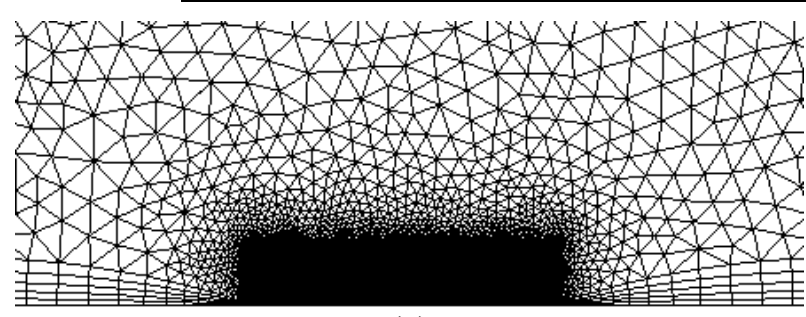

(a)

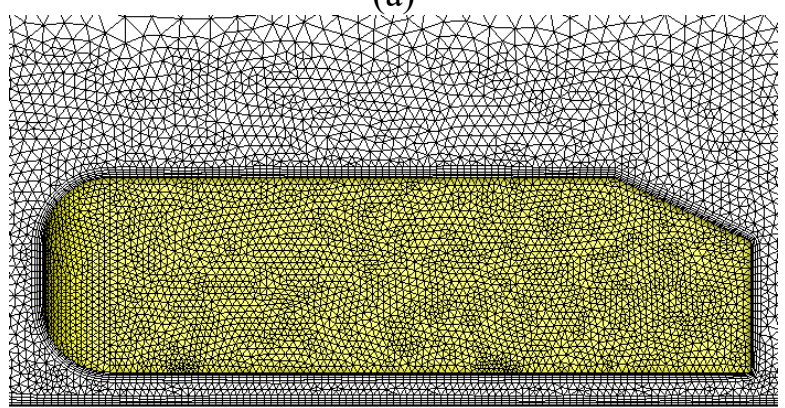

(c)

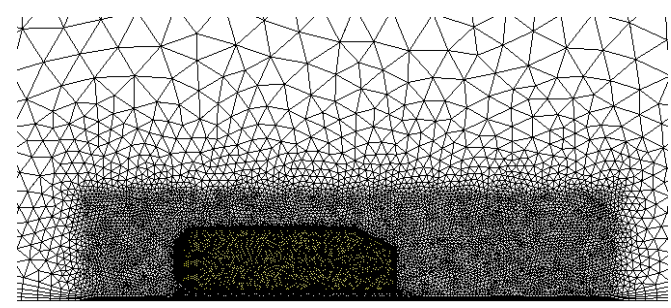

(b)

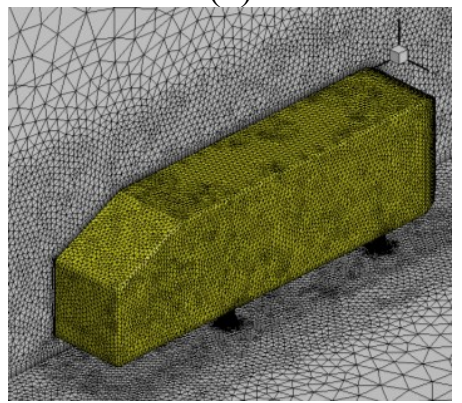

(d)

Figure 4 Applied mesh applied to the a) computational domain, b) small rectangular box around the body, c) boundary layer d) surface of the body.

Resolution of the mesh is represented by the non-dimensional wall coordinate of the first grid point. This is given by the nondimensional grid spacing $\mathrm{y}^{+}$. It is defined as

$$
y^{+}=\frac{y u^{*}}{v}
$$

Non-dimensional wall coordinate is a function of the wall friction velocity and is the molecular viscosity it, hence, depends on the local flow behavior [13]. As shown in Fig.5 the $\mathrm{y}^{+}$values are mostly in the range of $60 \sim 120$, therefore, the near wall treatment is applied to. It was reported that $30<\mathrm{y}^{+}<400$ is guarantee to work in turbulent sub-layer. The detailed knowledge on $\mathrm{y}^{+}$can be found in the ref. [14]. In the present study, realizable $\mathrm{k}-\varepsilon$ (RKE) turbulence is used for the simulations due to its suitability for complex shear flows involving

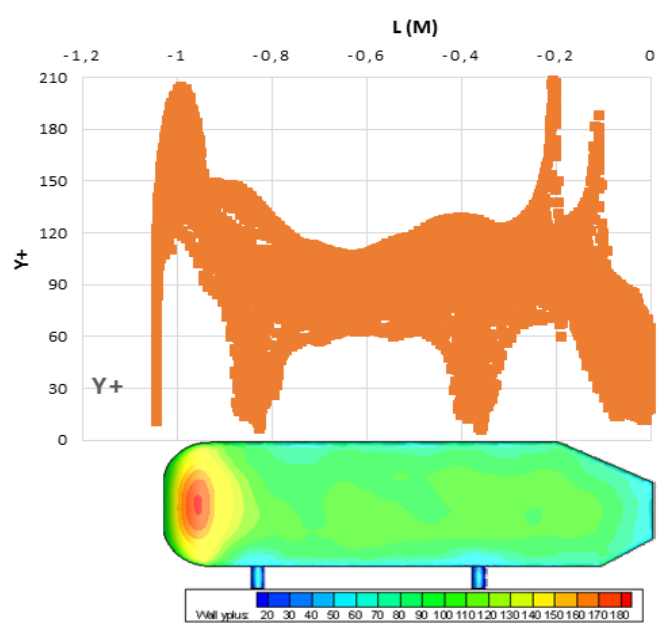

rapid strain, moderate swirl, vortices, and locally transitional flows such as boundary layer separation, massive separation, and Figure 5 Non-dimensional grid distance $\left(\mathrm{y}^{+}\right)$distribution on the body.

vortex shedding behind bluff bodies, stall in wide-angle diffusers [15]. Effects of some non- 
dimensional numbers such as Re number, $C_{D}$ and $C_{\mathrm{L}}$ are calculated as shown in Eq. 2-Eq.4, respectively.

$$
\begin{aligned}
& \mathrm{Re}=\frac{u_{\infty} L}{v} \\
& \frac{F_{D}}{\left(A_{p}\right)_{x}}=C_{D} \frac{\rho u_{\infty}{ }^{2}}{2} \\
& \frac{F_{L}}{\left(A_{p}\right)_{z}}=C_{L} \frac{\rho u_{\infty}{ }^{2}}{2}
\end{aligned}
$$

The convective and diffusion terms in the momentum, turbulent kinetic energy and its dissipation rate are discretized by the secondorder upwind scheme while pressure term is discretized by standard scheme. Pressurevelocity is coupled during the solutions.

In the present numerical study, effects of diffuser angle on the aerodynamic properties of the Ahmed body such as drag and lift coefficients are investigated for various upswept angles of the diffuser. Seven different upswept angles $(\beta)$ are taken into account; $\beta=0^{\circ}, 5^{\circ}, 10^{\circ}, 15^{\circ}, 20^{\circ}, 25^{\circ}$ and $30^{\circ}$ for a fixed slant angle $(\alpha)$ of $25^{\circ}$.

\section{Results and Discussion}

Validations of the present study are achieved by comparing results with the experimental data of [16], [17], and [18] in terms of the drag force and drag and lift coefficients. Change of stream-wise and normal components of velocity is also compared with [19] and [20]. As it is presented in Table 1, the drag coefficient is estimated with only $0.3 \%$ error. Although the lift coefficient deviates from the data of [17] by $4.3 \%$, it quite matches well with $\mathrm{C}_{\mathrm{L}}$ found by [18].

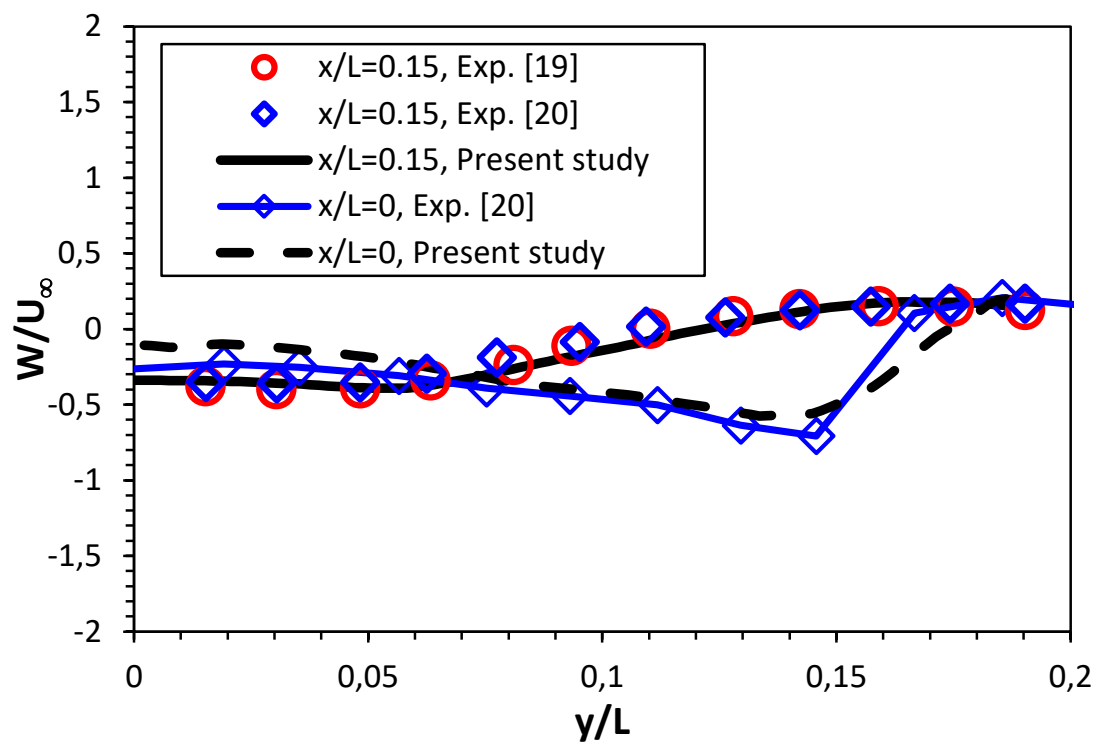

Figure 6 Span-wise velocity component comparison of the present study with [19] (red o) and [20] (blue $\diamond)$ for $\alpha=25^{\circ}$, $\beta=0^{\circ}$ at $\mathrm{z} / \mathrm{L}=0$ and $0.15, \mathrm{Re}=2.85 \times 10^{6}$.

Table 1. Compared values for slant angle of $\alpha=25^{\circ}$ at $\mathrm{Re}=2.85 \times 10^{6}$ (without diffuser).

\begin{tabular}{lccc}
\hline & $\mathrm{C}_{\mathrm{D}}{ }^{16}$ & $\mathrm{C}_{\mathrm{L}}{ }^{17}$ & $\mathrm{C}_{\mathrm{L}}{ }^{18}$ \\
& {$[-]$} & {$[-]$} & {$[-]$} \\
\hline Literature & 0.298 & 0.345 & 0.368 \\
Present study & 0.299 & 0.360 & 0.360 \\
Error (\%) & 0.3 & 4.3 & 2.1 \\
\hline
\end{tabular}

Stream-wise and span-wise velocity distributions are plotted against the data of [19] and [20] that obtained at two different streamwise stations; $\mathrm{x} / \mathrm{L}=0$ and 0.15 (Fig.6) and spanwise station of $\mathrm{z} / \mathrm{L}=0.26$ (Fig.7). It is seen that the present study is in good agreement with the data of [19] and [20] in terms of span-wise velocity component particularly at $\mathrm{x} / \mathrm{L}=0.15$. Due to change of the flow field after the body, it deviates a bit from data of [20], however, it agrees well in the far field regions.

Another comparison shown in Fig.7 shows that span-wise velocity component is better predicted compared with the stream-wise velocity component due to using coarser mesh toward that station. The diffuser generated by upsweeping the rear bottom of the body can be described as a passage of increasing area that is used to reduce the velocity of the flow. 


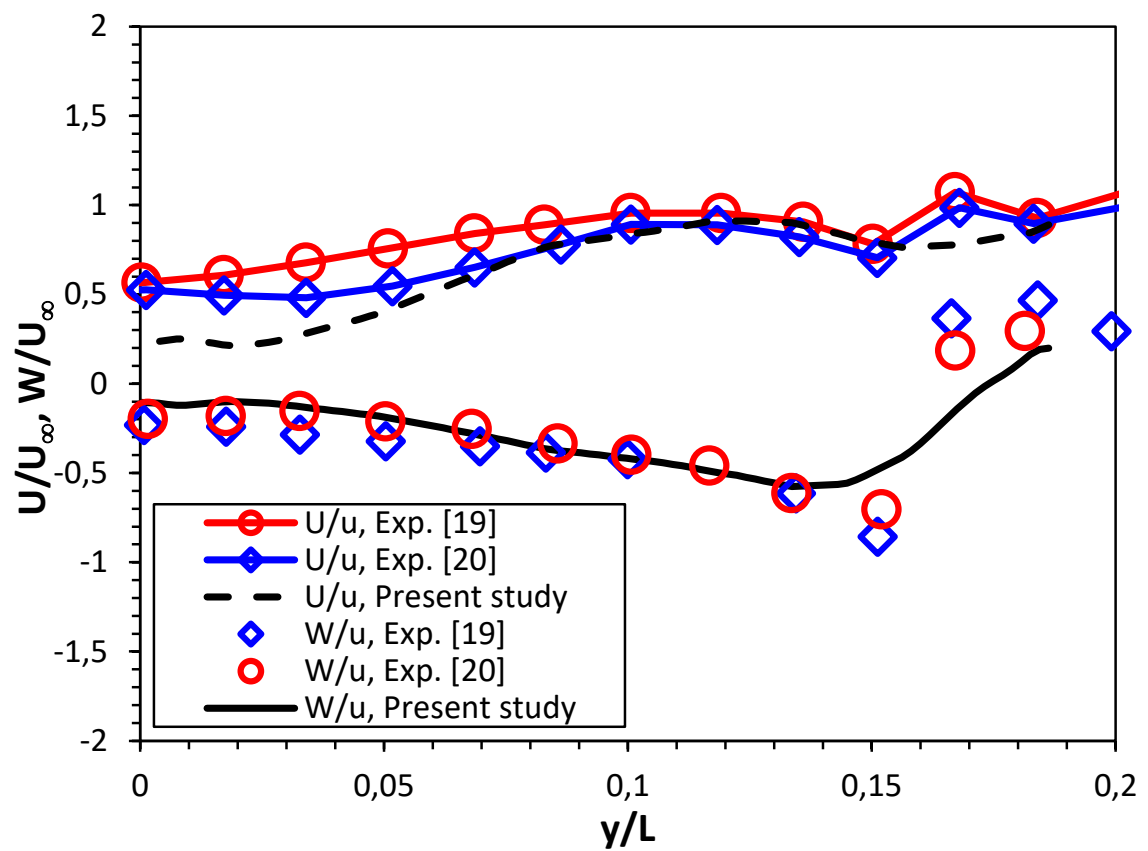

Figure 7 Stream-wise and normal velocity components comparison of the present study with [19] (red o) and [20] (blue $\diamond$ ) for $\alpha=25^{\circ}, \beta=0^{\circ}$ at $\mathrm{z} / \mathrm{L}=0.26, \mathrm{Re}=2.85 \times 10^{6}$.

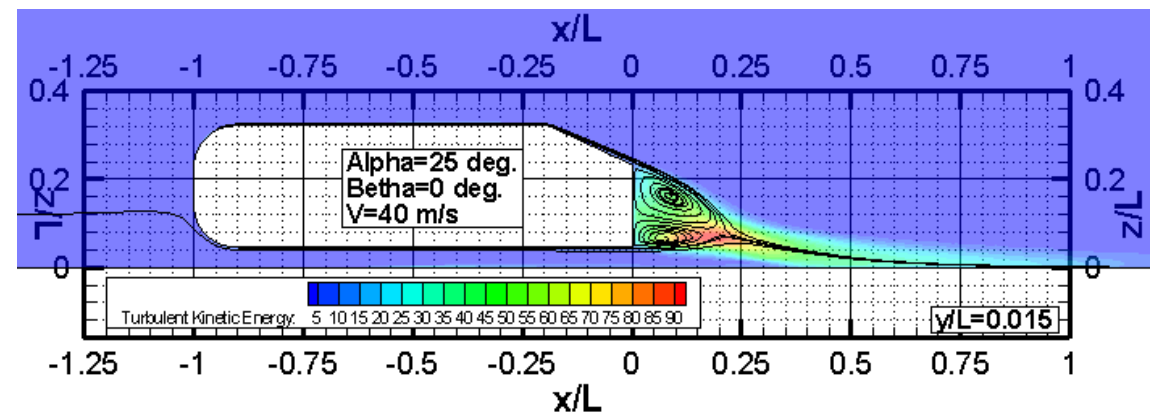

(a)

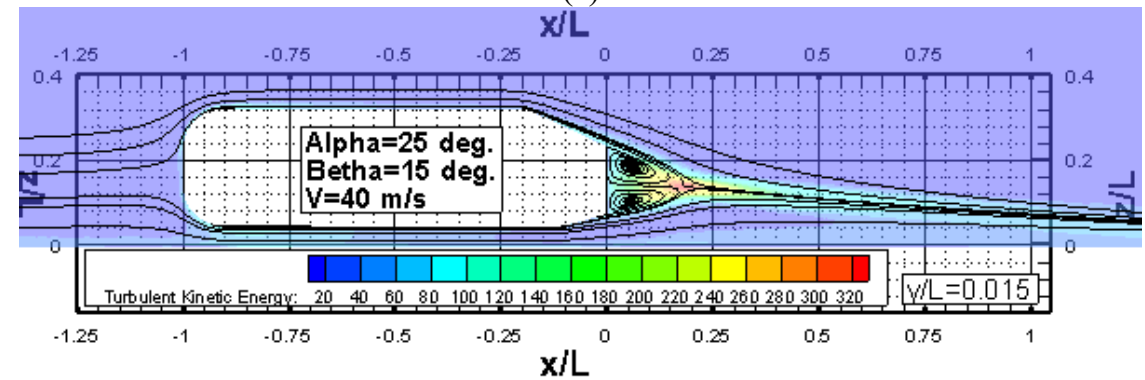

(b)

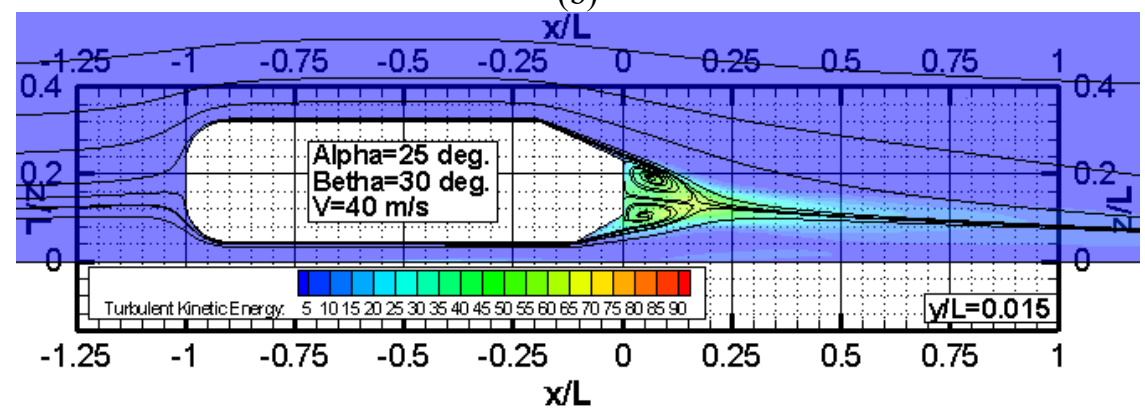

(c)

Figure 8 Turbulence kinetic energy contours and streamlines behind body when the diffusor angle is a) $\beta=0^{\circ}$, b) $\beta=15^{\circ}$ and c) $\beta=30^{\circ}$ at $\mathrm{y} / \mathrm{L}=0.015$ at $\mathrm{Re}=2.85 \times 10^{6}$.

Streamlines and the turbulence kinetic energy distributions due to the existence of such passage are shown in Fig.8. It is seen that flow reversal zone behind the body changes with the 
upswept angle. As it increases from $\beta=0^{\circ}$ to $30^{\circ}$ flow reversal zone moves upward and the two contra-rotating bubbles become almost symmetrical. The turbulent kinetic energy contours show that the maximum turbulent kinetic energy is located around the lowest separation bubble in the wake behind the body and when the upswept angle increases the maximum turbulent kinetic energy moves upward because the boundary layers coming from the roof and the diffuser merge in the approximately at the center of the reversal zone. Stream-wise velocity contours and the flow field behind the body for various upswept angles are shown in Fig. 9 and 10. As presented in Fig. 9 at the first station a counterrotating vortex pair starts to form on each side of the body just behind it and starts to grow in both directions when the upswept angle is $\beta=0^{\circ}$. Such vortex structure is titled as the Cpillar vortex in the literature [21]. However, when the upswept angle increases to $15^{\circ}$ and $30^{\circ}$ two rotating vortex pair occur beneath the

$\beta$
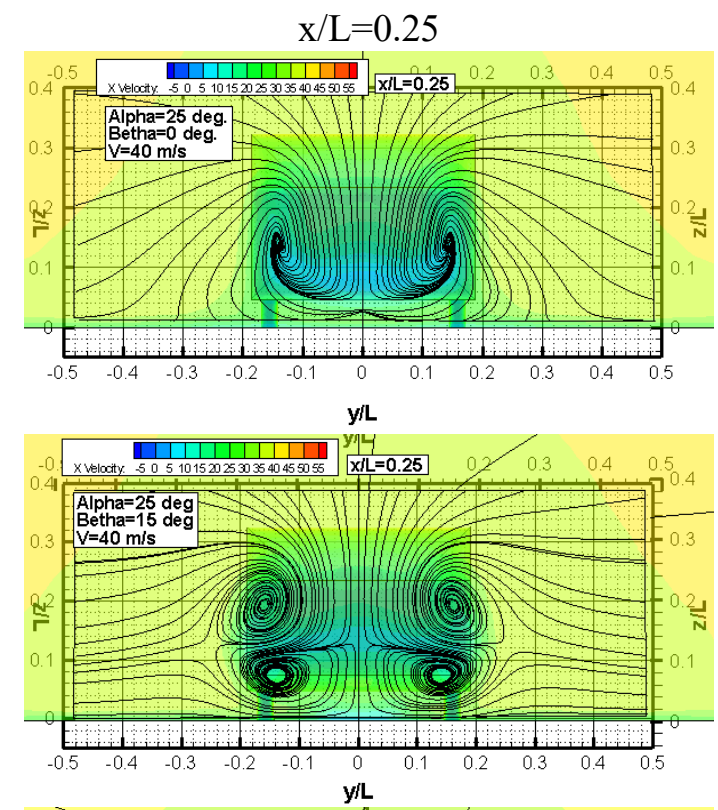

$15^{\circ}$

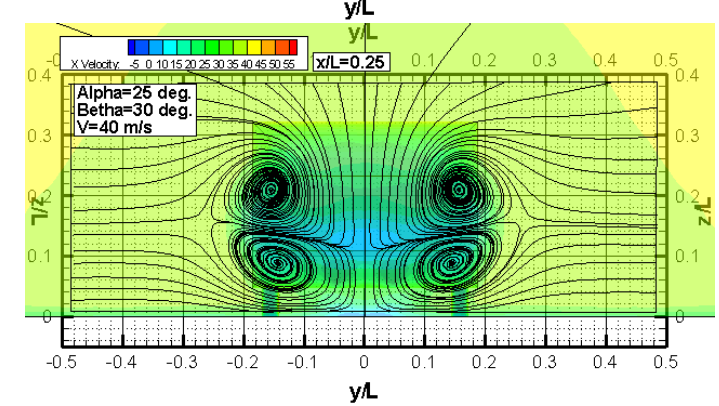

C-pillar vortices in each side of the body. In the subsequent stations, these vortices merge with the C-pillar vortices, and grow while disappearing completely at $\mathrm{x} / \mathrm{L}=2.0$ as shown in Fig.10. These vortices originate from the upswept section called diffuser of the body, span along the lateral directions due to low pressure in the upswept section.

A vortex is defined as a circular or spiral set of streamlines while a vortex core is a special type of iso-surface that displays a vortex or the rotation motion of a multitude of material particles around a common center [22]. Fig. 11 and 12 show the vortex systems such as Cpillar vortex, corner vortex and vortex behind the body. As can be seen in Fig. 11 increasing velocity does not affect the structure of the vortex systems. However, when the upswept angle increases the second vortex behind the diffuser thickens and merges with the vortex system wrapped the lower end of the body (Fig.12).
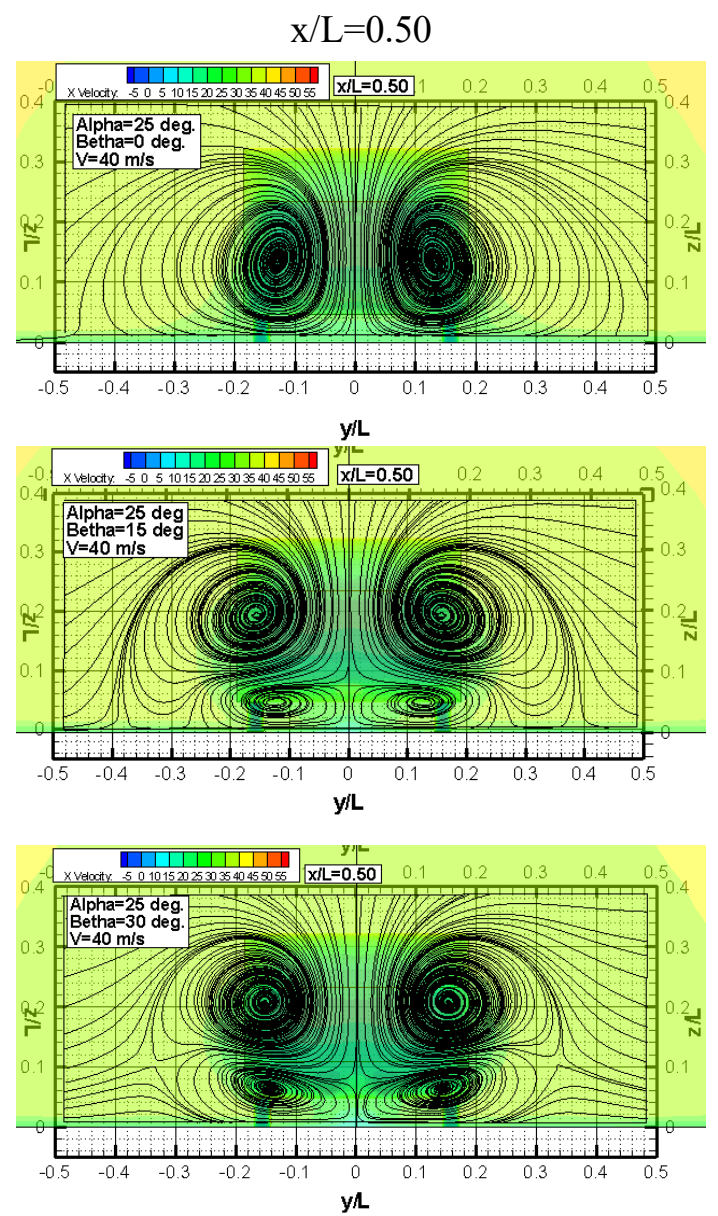

Figure 9 Change of streamwise velocity and flow field behind the body at $\mathrm{x} / \mathrm{L}=0.25$ and 0.50 for various upswept angles. 
$\beta$

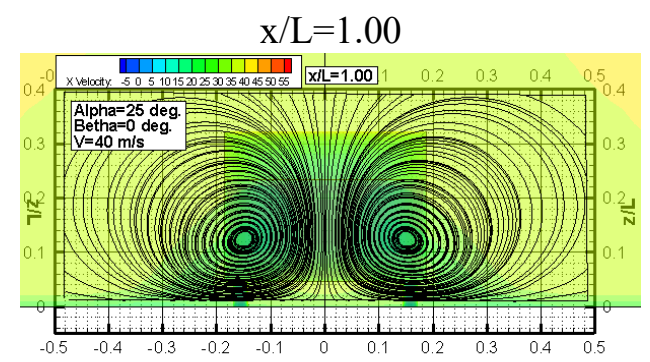

$15^{\circ}$

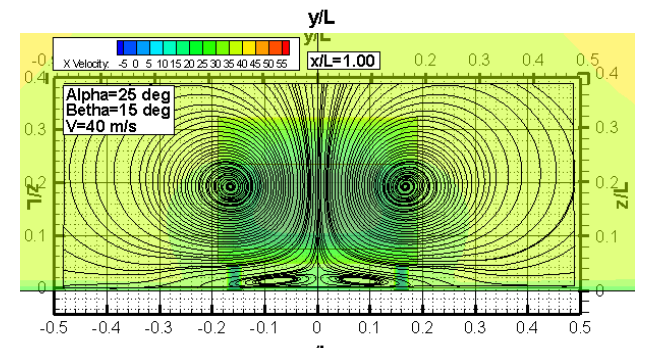

yiL

$30^{\circ}$

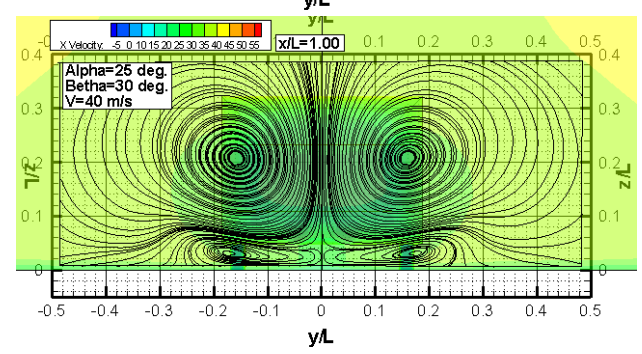

$\mathrm{x} / \mathrm{L}=2.00$

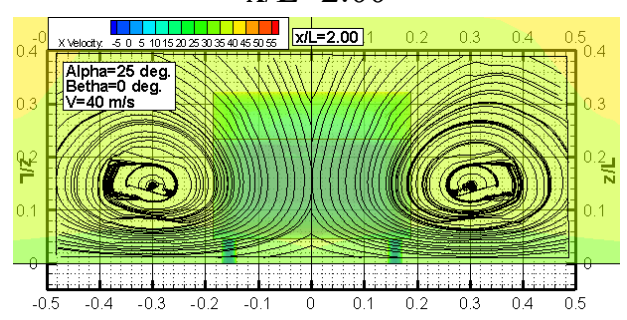

y/L

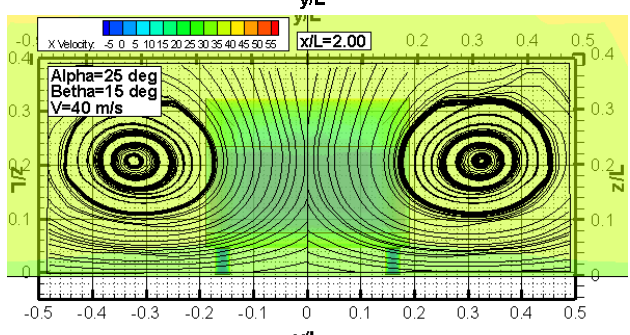

yil

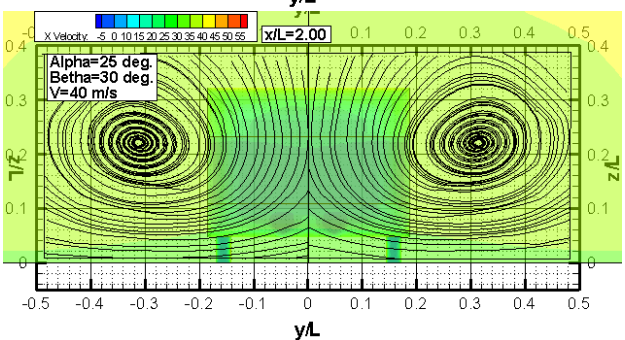

Figure 10 Change of streamwise velocity and flow field behind the body at $x / L=1.00$ and 2.00 for various upswept angles.
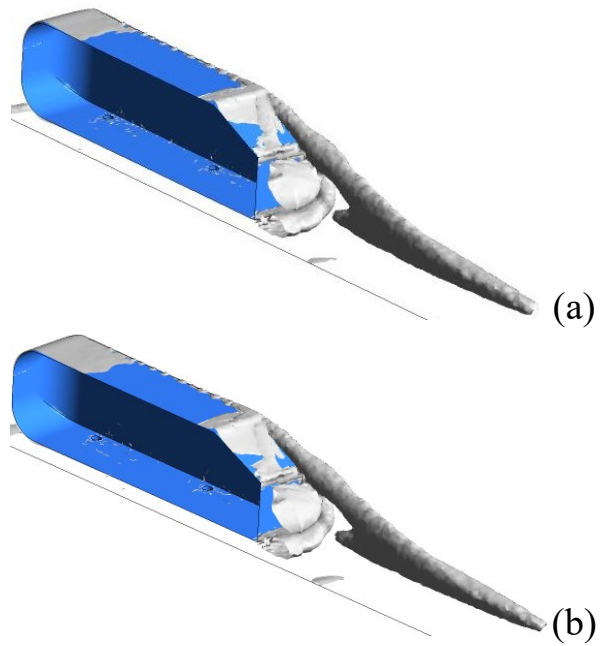

Figure 11 Vortex core region behind the Ahmed body at a) $\mathrm{V}=10 \mathrm{~m} / \mathrm{s}, \mathrm{b}) \mathrm{V}=40 \mathrm{~m} / \mathrm{s},\left(\beta=0^{\circ}\right.$, level $\left.=0.0051 / \mathrm{s}\right)$

Two most important aerodynamic parameters are the drag coefficient that quantifies the advancing resistance posed by surrounding fluid onto the vehicle and the lift coefficient that represents the force exerted onto the vehicle in the direction perpendicular to the flow. In the aerodynamics of the ground vehicles reducing drag coefficient is very important because it reduces the overall fuel consumption of the vehicle. On the other hand, reducing the lift coefficient will improve ride stability and cornering performance. Change of drag coefficient with the various upswept angles and the free-stream velocities are plotted in Fig. 13. It is seen that regardless of the upswept angle drag coefficient reduces as the free-stream velocity increases. Increasing the diffuser angle reduces the drag coefficient until $\beta=15^{\circ}$ and after that drag coefficient increases gradually and reaches its maximum.

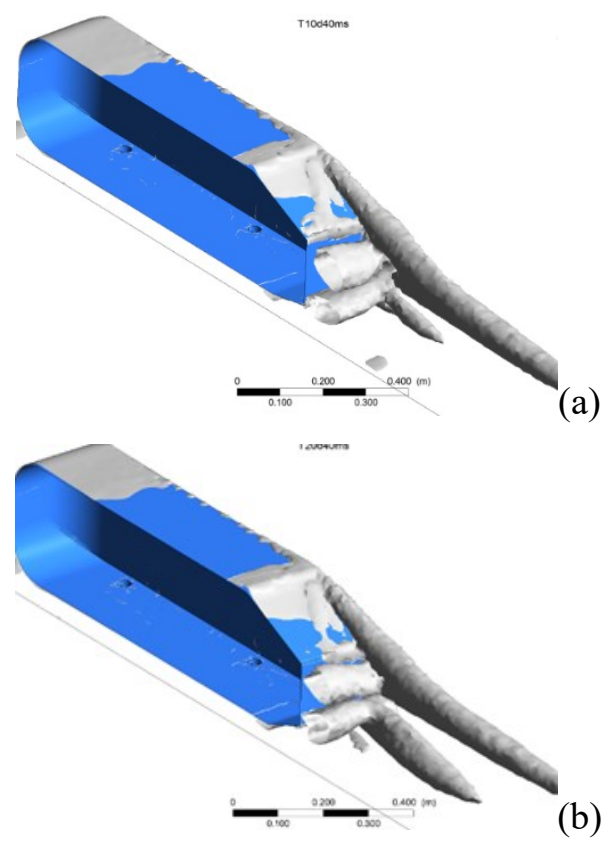

Figure 12 Vortex core region behind the Ahmed body for a) $\beta=10^{\circ}$, b) $\beta=20^{\circ}(\mathrm{V}=40 \mathrm{~m} / \mathrm{s}$, level $=0.0051 / \mathrm{s})$ 


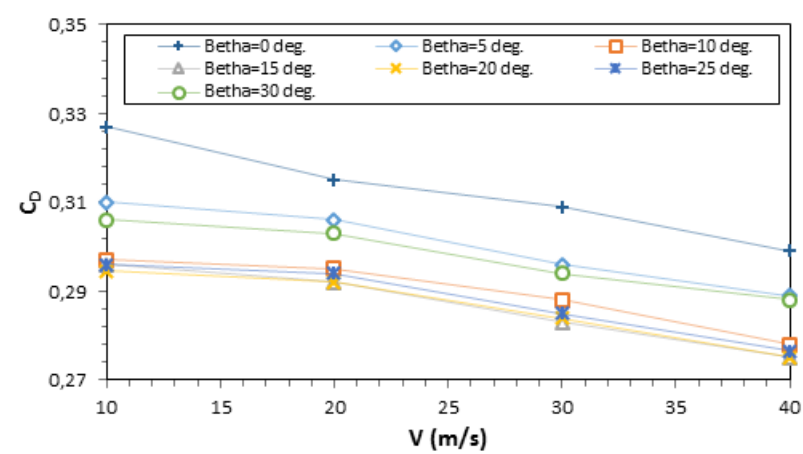

(a)

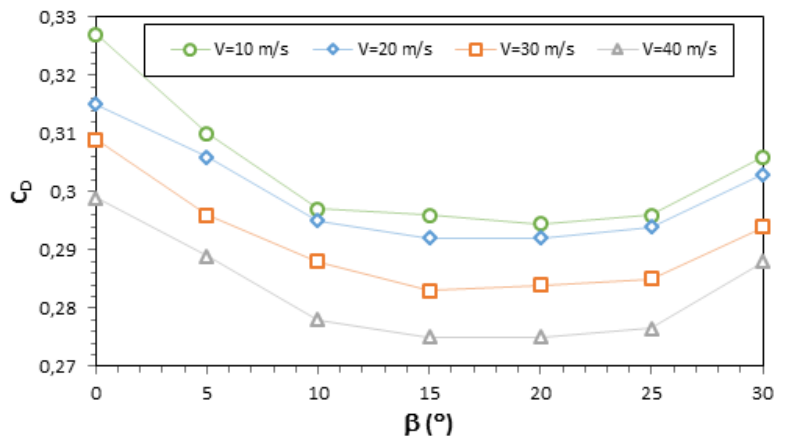

(b)

Figure 13 Drag coefficient distributions for various a) upswept angle and b) velocities.

It is demonstrated by several researchers that a symmetrical body in a free stream has zero lift coefficient when the side walls of the computational domain are far away from the cylinder [23], however, as the cylinder is moved toward one of the side walls the flow along the underside of the cylinder is constrained and causing flow acceleration and resulting decrease in static pressure. Therefore, it is not surprise to see a suction on the underbody. Such low pressure underneath the body generates a downforce on the body and increase grip. Such effects of velocity and diffuser angle on the lift coefficient are presented in Fig. 14. Although the similar trends are observed as for drag coefficient, the most critical upswept angle is $\beta=20^{\circ}$ in case of lift coefficient because lift coefficient reduces almost linearly to this angle and increases with the upswept angle. The existence of the upswept section causes the flow accelerates over the bottom surface of the body resulting low pressure and accordingly increases the downforce over the body due to the difference in the acceleration of the flow between the top and bottom surfaces of the body [12]. Such differences increases with the higher upswept angles and therefore higher upswept angles provide lower lift coefficients. It is seen that the critical upswept angle is $\beta=20^{\circ}$ since it provides the negative lift coefficients at higher free-stream velocities ( $\mathrm{V}=30 \mathrm{~m} / \mathrm{s}$ and $40 \mathrm{~m} / \mathrm{s}$ ).It is known that the lower lift coefficients enhance the overall grip of the vehicle while higher lift coefficients are not desired for ground vehicles. From this perspective, it can be concluded that the optimum upswept angle is the $\beta=20^{\circ}$ for these cases.

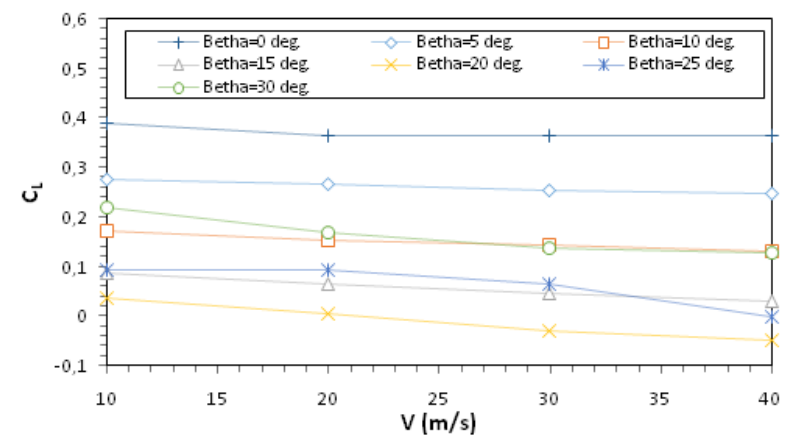

(a)

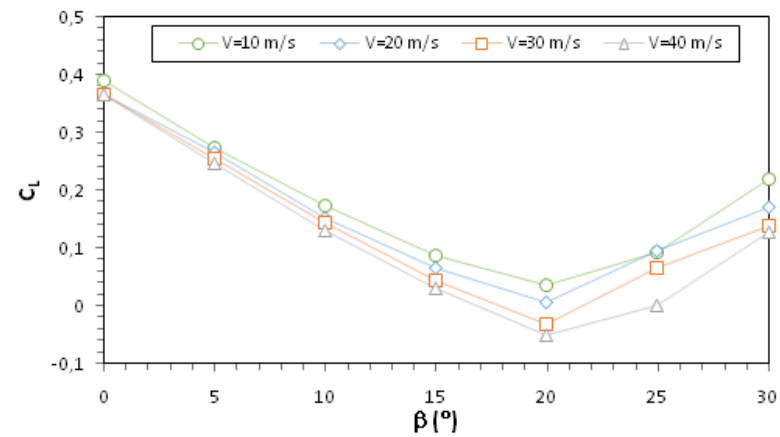

(b)

Figure 14Lift coefficient distributions for various a) upswept angle and b) velocities.

Pressure coefficient variation over the top and bottom surfaces of the modified Ahmed body is shown in Fig.15. It is seen that the pressure coefficient at the stagnation point at the front end of the body remained constant at $C_{p}=1$. As the fluid flow over the sides of the body its velocity increases while the pressure decreases at these regions resulting decreases in the pressure coefficient at $-1<\mathrm{x} / \mathrm{L}<-0.9$. When it flows over the straight top and bottom surfaces the pressure recoveries and changes a little bit after the struts. This is why the pressure coefficient distribution over the bottom surface deviates from the top surface at $-0.8<\mathrm{x} / \mathrm{L}<-0.6$. Since the existence of the upswept section has not affected the flow yet the pressure coefficient distribution is the same for all upswept angles in the region $-1<\mathrm{x} / \mathrm{L}<-0.10$. It is seen that the pressure coefficient over both surfaces drops dramatically at $\mathrm{x} / \mathrm{L}=-0.2$ due to 
the slanted back of the body at the rear. Another sudden drop is seen at $\mathrm{x} / \mathrm{L}=-0.10$ due to the various configuration of the upswept section. The lowest pressure coefficient is obtained for $\beta=20^{\circ}$ which provides the lowest lift coefficient and provides the maximum grip.

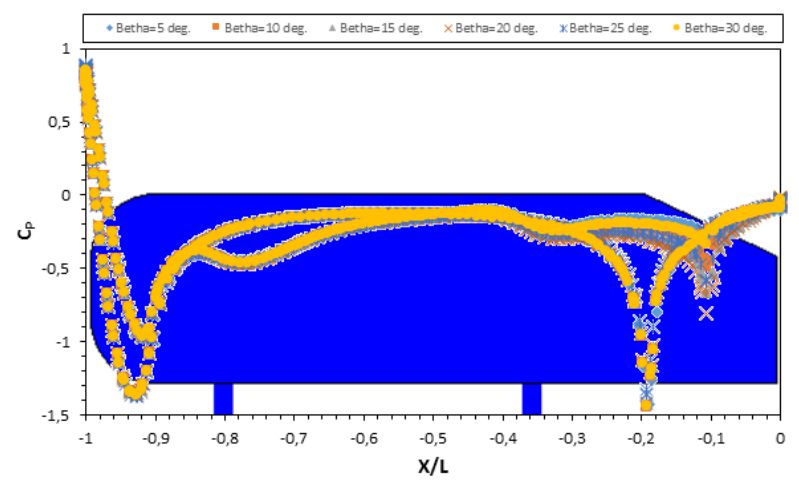

(a)

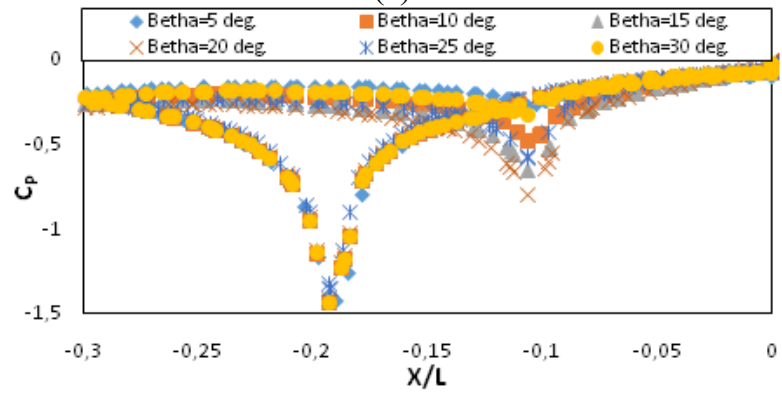

(b)

Figure 15Pressure coefficient distribution over the body (a) and the close-up view of it at the rear of the body, $0.71 \times 10^{6}$.

\section{Conclusions}

In the present study, effects of a diffuser obtained by upsweeping the rear part of generic Ahmed body are investigated numerically for various Re numbers and upswept angles. It is determined that the reversal zone behind the upswept body changes with its angle and moves upward. Turbulent kinetic energy densify toward the upper zones since boundary layers coming from the roof and the diffuser merge in the approximately at the center of the reversal zone. In comparison with the counterrotating vortex pair seen behind the Ahmed body without diffuser two rotating vortex pair form beneath the so-called C-pillar vortices. Although $\mathrm{Re}$ number does not affect the structure of the vortex systems the existence of the diffuser changes the strength of them. Study on the effects of the diffuser shows that drag coefficient reduces with increasing $\mathrm{Re}$ number at any upswept angle. Among several upswept angles, the critical one is found as to be $15^{\circ}$ because drag coefficient reduces sharply up to $15^{\circ}$ and then increases. Lift coefficient also decreases with increasing $\mathrm{Re}$ number. However, unlike the change in the drag coefficient it changes slightly. It seen that the critical lift coefficient is $20^{\circ}$.

\section{Nomenclature}

$\begin{array}{ll}A_{P} & \text { Projected frontal area of Ahmed body } \\ C_{D} & \text { Drag coefficient } \\ C_{\mathrm{L}} & \text { Lift coefficient } \\ \mathrm{C}_{\mathrm{p}} & \text { Pressure coefficient } \\ \mathrm{CFD} & \text { Computational fluid dynamics } \\ \mathrm{L} & \text { Length of Ahmed body } \\ \mathrm{Re} & \text { Reynolds number } \\ \mathrm{u}_{\infty} & \text { Freestream velocity } \\ \mathrm{u}^{*} & \text { Wall friction velocity } \\ \mathrm{x} & \text { Streamwise direction } \\ \mathrm{y} & \text { Normal direction } \\ 3 \mathrm{D} & \text { Three-dimensional } \\ \mathrm{y}^{+} & \text {Non-dimensional grid spacing } \\ \alpha & \text { Slant angle of Ahmed body } \\ \rho & \text { Density of the fluid } \\ \beta & \text { Upswept angles of Ahmed body } \\ v & \text { Molecular viscosity }\end{array}$

\section{References}

1. Hucho, W., Sovran, W.H., Aerodynamics of road vehicles, Annual Review of Fluid Mechanics, 25, 485-537, 1993 2. Kang, S.O., Jun, S.O., Park, H.I., Song, K.S., Kee, J.D., Kim, K.H., Lee, D.., Actively translating a rear diffuser device for the aerodynamic drag reduction of a passenger car, International Journal of Automotive Technology, 13(4), 583-592, 2012.

3. Ahmed, S.R., Ramm, G., Faltin, G., Some salient features of the time-averaged ground vehicle, SAE Technical paper 840300 , 1-30, Society of Automotive Engineers, inc, Warrendale, PA, USA, 1984.

4. Franck, G., Nigro, N., Storti, M., Delia, J., Numerical simulation of the flow around the Ahmed vehicle body, Latin American Applied Research, 39, 295-306, 2009.

5. Beaudoin, J.F., Aider, J.L., Drag and lift reduction of a 3D bluff body using flaps, Experiments in Fluids, 44, 491-501, 2008.

6. Gohlke, M., Beaudoin, J.f., Amieh, M., Anselmet, F., Shape influence on mean forces applied on a ground vehicle under steady cross- 
wind, Journal of Wind Engineering and Industrial Aerodynamics, 98, 386-391, 2010.

7. Aider, J.L., Beaudoin, J.F., Wesfreid, J.E., Drag and lift reduction of a 3D bluff body using active vortex generators, Experiments in Fluids, 48, 771-789, 2010.

8. Fourrie, G., Keirsbulck, L., Labraga, L., Gillieron, P., Bluff-body drag reduction using a deflector, Experiments in Fluids, 50, 385-395, 2011.

9. Joseph, P., Amandolese, X., Aider, J.L., Drag reduction on the $25^{\circ}$ slant angle Ahmed reference body using pulsed jets, Experiments in Fluids, 52, 1169-1185, 2012.

10. Krajnovic, S., Large eddy simulation exploration of passive flow control around an Ahmed body, Trans. ASME: J. Fluid Engng., 136, 121103, 2014.

11. Pujals, G., Depardon, S., Cossu, C., Drag reduction of a $3 \mathrm{D}$ bluff body using coherent streamwise streaks, Experiments in Fluids, 49, 1085-1094, 2010.

12. Zhang, X., Senior, A., Ruhrmann, A., Vortices Behind a Bluff Body with an Upswept Aft Section in Ground Effect, International journal of Heat and Fluid Flow, 25, 1-9, 2004.

13. Howard, R.J.A., Pourquie, M., Large eddy simulation of an Ahmed reference model, Journal of Turbulence, 3, n12, 2002.

14. Andersson, B., Andersson, R., Hakansson, L., Mortensen, M., Sudiyo, R., Van Wachem, B., Computational Fluid Dynamics for Engineers, Cambridge University Press, 2012.

15. Ansys Fluent 12.0 User's Guide, 2012

16. Bayraktar, I., Landman, D., Baysal, O., Experimental and Computational Investigation of Ahmed Body for Ground Vehicle Aerodynamics, SAE paper 2001-01-2742, 2001.

17. Graysmith J.L., Baxendale A.J., Howell J.P., Haynes T., Comparisons between CFD and experimental results for the Ahmed reference model. RAeS conference on vehicle aerodynamics, Loughborough, pp 30.1-30.11, 1994.

18. Muritala, A.O., Fatokun, H.A, Obayopo, S.O., Effect of an Add-On Device on the Aerodynamic Characteristics of a 3Dimensional Ahmed Boyd, IOSR Journal of Mechanical and Civil Engineering (IOSRJMCE), 14(6), 18-29, 2017.
19. Lienhart, H., Stoots, C., Becker, S., Flow and Turbulence in the Wake of a Simplified Car Model (Ahmed Model). In Wagner, S., Rist, U., Heinemann, H.J., Hilbig, R. (ed.), New Results in Numerical and Experimental Fluid Mechanics III. Notes on Numerical Fluid Mechanics (NNFM), vol.77, Springer, Berlin. Heidelberg, 2002.

20. Strachan, R.K., Knowles, K., Lawson, N.J., The vortex structure behind an Ahmed reference model in the presence of a moving ground plane, Exp. Fluids, 42, 659-669, 2007.

21. Keogh, J., Barber, T., Diasinos, S., Doig, G., The Aerodynamic Effects on a Cornering Ahmed Body, J. of Wind Eng. Ind. Aerodyn., 154, 34-46, 2016.

22. Ming, J., Raghu, M., David, T., Detection and Visualization of Vortices. The visual handbook. 10.1016/B978-0123875822/50016-2, 2005

23. Bayraktar, S., Yayla, S., Oztekin, A., Ma, H. Wall Proximity Effects on Flow over Cylinders with Different Cross Sections, Canadian Journal of Physics, 92, 141-148, 2014. 